\title{
Development of a tele-iaudiometer system
}

\begin{abstract}
According to the World health Organization1, “over 5\% of the world's population 360 million people - has a disabling hearing loss (328million adults and 32million children). Disabling hearing loss refers to hearing loss greater than 40 decibels $(\mathrm{dB})$ in the better hearing ear in adults and a hearing loss greater than $30 \mathrm{~dB}$ in the better hearing ear in children. The majority of people with disabling hearing loss live in low- and middle-income countries.

Approximately one-third of people over 65years of age are affected by disabling hearing loss. The prevalence in this age group is greatest in South Asia, Asia Pacific and subSaharan Africa."

Hearing loss affects the individual's ability to communicate with others. Spoken language development is often delayed in children with deafness. Ear diseases such as otitis media can contribute a significantly adverse effect on the academic ability of children to learn. In developing countries, children with hearing loss and deafness rarely receive an education. There is also a much higher unemployment rate among adults with a hearing impairment. Those adults with a hearing impairment (who are employed) have a higher percentage of jobs in the lower levels of employment compared with the general hearing population. Improving access to education and vocational rehabilitation services, and raising awareness especially among employers about the needs of people with hearing loss, would decrease unemployment rates among this group. In addition to the economic impact of hearing loss there is also a substantial effect on the social and economic development in communities and countries. Hearing healthcare for individuals living in third world countries is often either not available, or there is limited access. Often times, early intervention for acute ear problems can prevent serious or even life threatening ear diseases. Early diagnosis and treatment are essential. What is desperately needed is the availability of a cost effective hearing healthcare delivery system.
\end{abstract}

Keywords: audiometer, hearing impairment, hearing loss, hearing evaluation, audiometer $^{\mathrm{TM}}$, tele-iaudiometer ${ }^{\mathrm{TM}}$, hearing healthcare
Volume 6 Issue 6 - 2017

\author{
David W Holmes, ' Antonio Curci, ${ }^{2}$ Gianna \\ Lardaro, ${ }^{2}$ Marshall Wenrich' \\ 'Melmedtronics, USA \\ Melmedtronics, Italy
}

Correspondence: David W Holmes Melmedtronics 2083 Rana Park Flint TX 75762, USA, Tel +|-8| 7-20|-679|,

Email drdavidholmes@melmedtronics.com

Received: November 07, 2016 | Published: April 18, 2017

\section{Introduction}

The evaluation of hearing is critical to the diagnosis and management of someone presenting with the complaint(s) of ear pain, loss of hearing, tinnitus and/or dizziness or vertigo. If you are fortunate to live in a country with a first class healthcare system, then you are in a select group who will no doubt have access to early diagnosis, intervention and successful treatment. However, there are still many individuals, even in first-world countries, who still have limited access to quality hearing healthcare. ${ }^{2}$ Some of the reasons can be attributable to living in rural areas, lack of transportation, too ill to travel, other concomitant conditions, or local healthcare facilities do not have the specialized equipment, or trained personnel to use the equipment. So, even first-world societies often share the similar lack of hearing healthcare access that is found in third-world countries for segments of their own population.

The solution to this dilemma is the development of a hearing healthcare delivery system that is:
A. Cost effective
B. Easily administered
C. Readily available
D. Deployed to any location

We have developed a system that meets all of these requirements. We have developed an audiometer that uses an iPad and/or an iPhone as the device (Figure 1). It uses the same transducers as a traditional audiometer, delivers the same auditory tests, and is calibrated to ANSI S3.6:2010 3 and ISO IEC EN 60645-2002-07 Type $2^{4}$ Standards.

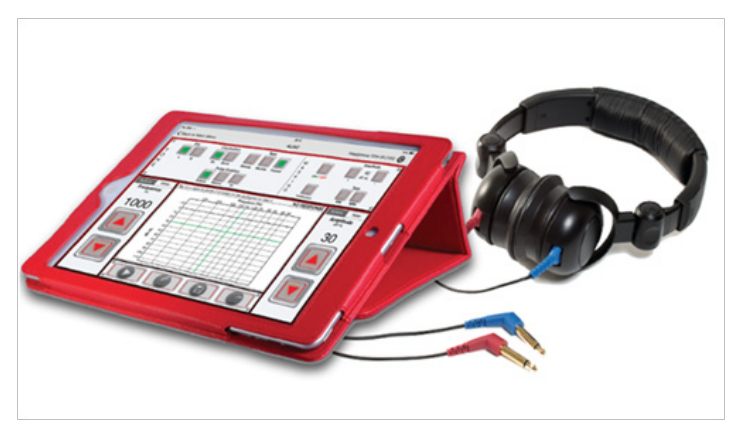

Figure I iPad Audiometer with DD45 Headphones.

Because of the use of a mobile platform it is now possible to remotely evaluate a patient anywhere in the world using either a WiFi or cellular service (Figure 2).

Other advantages include the ability to have real-time audio and video back and forth communication between the Clinician's site and the Patient's site (Figure 3). Test results can also be saved, emailed, printed and integrated into any EHR system.

Both the iAudiometer and Tele-iAudiometer systems use a 256 AES encryption, a 0.5 Mbps bandwidth and they are all HIPAA compliant (Figure 4). 


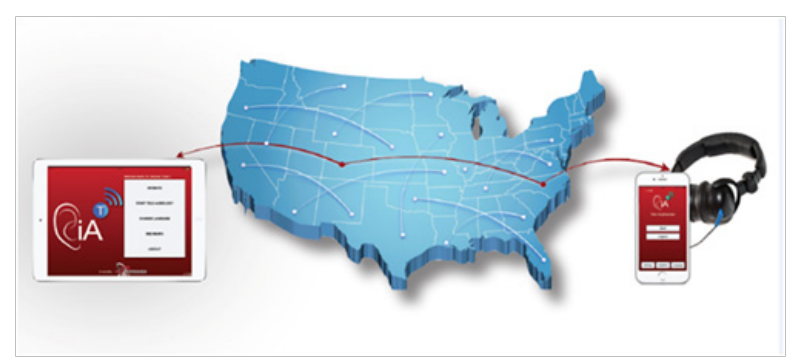

Figure 2 Example of a Worldwide Connection.

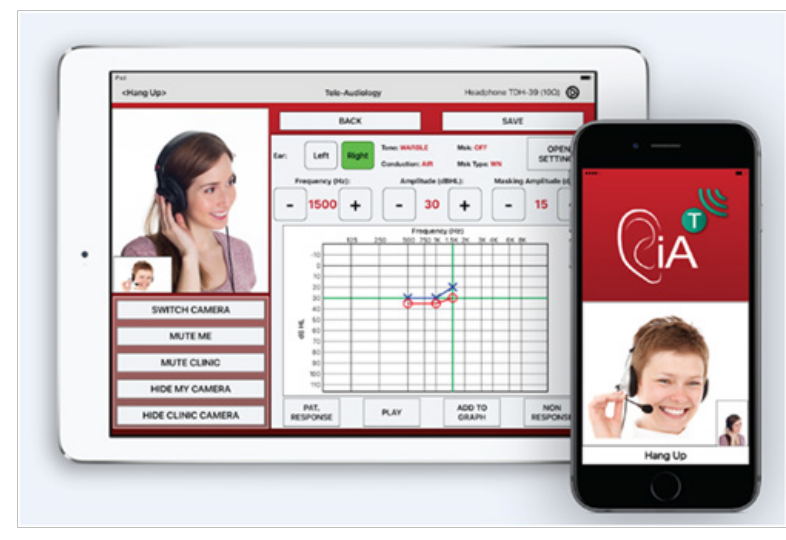

Figure 3 Patient's Site Device.

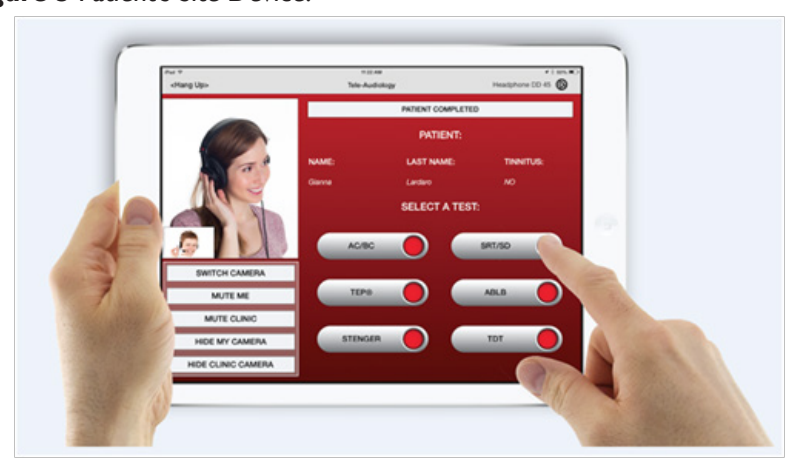

Figure 4 Clinician’s Site Device.

\section{Conclusion}

The use of mobile medical devices to evaluate hearing remotely is an economical solution to reach millions of individuals who could not otherwise be evaluated, diagnosed and treated in a timely manner.

\section{Acknowledgements}

The authors would like to thank Eugenio Luciana, Diego Fogliata and Marshall Wenrich for their significant contributions to the development of these systems.

\section{Conflicts of interest}

Dr. David Holmes, Dr. Antonio Cursi, Dr. Gianna Lardaro and Mr. Wenrich all have a financial interest in Melmedtronics.

\section{Funding}

None.

\section{References}

1. http://www.who.int/mediacentre/factsheets/fs300/en/

2. https://www.ruralhealthinfo.org/topics/rural-health-disparities

3. http://www.ncbi.nlm.nih.gov/pmc/articles/PMC3292602/

4. Robert H, Margolis and Samantha M. Stiepan, Acoustic method for calibration of audiometric bone vibrators. J Acoust Soc Am. 2012;131(2):1221-1225.

5. https://webstore.iec.ch/publication/2771

6. Electroacoustics - Audiometric equipment - Part 1: Equipment for puretone audiometry. 2012;IEC 60645-60651. 